\title{
Entanglement and Fidelity Signatures of Quantum Phase Transitions in Spin Liquid Models
}

\author{
Amit Tribedi and Indrani Bose \\ November 11, 2018 \\ Department of Physics \\ Bose Institute \\ 93/1, Acharya Prafulla Chandra Road \\ Kolkata - 700 009, India
}

\begin{abstract}
We consider a spin ladder model which is known to have matrix product states as exact ground states with spin liquid characteristics. The model has two critical-point transitions at the parameter values $u=0$ and $\infty$. We study the variation of entanglement and fidelity measures in the ground states as a function of $u$ and specially look for signatures of quantum phase transitions at $u=0$ and $\infty$. The two different entanglement measures used are $S(i)$ (the single-site von Neumann entropy) and $S(i, j)$ (the two-body entanglement). At the quantum critical point (QCP) $u=\infty$, the entanglement measure $E[=S(i), S(i, j)]$ vanishes but remains non-zero at the other QCP $u=0$. The first and second derivative of $E$ with respect to the parameter $u$ and the entanglement length associated with $S(i, j)$ are further calculated to identify special features, if any, near the QCPs. We further determine the GS fidelity $F$ and a quantity $\ln |D|$ related to the second derivative of $F$ and show that these quantities calculated for finite-sized systems are good indicators of QPTs occurring in the infinite system.
\end{abstract}

\section{INTRODUCTION}

In recent years, quantum phase transitions (QPTs) in many-particle systems have been extensively investigated using well-known quantum information theoretic measures. QPTs which are solely driven by quantum fluctuations occur at zero temperature when some parameter, either external or intrinsic to the Hamiltonian, is tuned to a special value termed the transition point [1]. In the case of second-order QPTs (critical-point transitions), a diverging length scale governs the physics near a quantum critical point (QCP). Usually, the correlation length associated with specific correlation functions diverges as the QCP is approached and the ground state properties develop non-analytic features. In this context, it is pertinent to ask whether the quantum correlations associated with entanglement are good indicators of QPTs. A number of entanglement measures have so far been identified which show special features close to the transition points of QPTs occuring in spin systems 
[2, 3, 4, 5, 6, 7]. It has been shown that, in general, a first order QPT, linked to a discontinuity in the first derivative of the ground state energy, is signalled by a discontinuity in a bipartite entanglement measure such as negativity and concurrence [8, 9, 10] and a discontinuity or a divergence in the first derivative of the same entanglement measure marks a second order phase transition characterized by a discontinuity or a divergence in the second derivative of the ground state energy [2, 3]. The entropy of entanglement of a block of $L$ contiguous spins in a chain with the rest of the system has been shown to diverge logarithmically with $L$ near the QCP [4].

The typical length scale over which a particular entanglement measure decays defines the entanglement length (EL). A number of entanglement measures characterized by a diverging EL close to a QCP have been proposed to date [7, 11]. One of these, the two-body entanglement $S(i, j)$ which estimates the amount of non-local correlations between a pair of separated spins at sites $i$ and $j$ and the rest of the spins, is given by the von Neumann entropy

$$
S(i, j)=-\operatorname{Tr} \rho(i, j) \log _{2} \rho(i, j)
$$

where $\rho(i, j)$ is the two-site reduced density matrix obtained by tracing out the spins except the ones at sites $i$ and $j$ from the full density matrix. When the system is translationally invariant, $S$ depends only on the separation $n=|j-i|$ and can be expressed in terms of the spin correlation functions in the large $n$ limit. Away from the critical point, $S(i, j)$ saturates over a length scale $\xi_{E}$, the EL, as $n$ increases. Close to the QCP and for large $n$, we have

$$
S(n)-S(\infty) \sim A(n) e^{-\frac{n}{\xi_{E}}}
$$

$S(i, j)$ captures the long-range correlations at a QCP if $A(n)$ has a power-law decay as a function of $n$ along with a divergent $\xi_{E}$. This is true for spin models such as the $S=\frac{1}{2}$ exactly solvable anisotropic XY model in a transverse magnetic field [7]. The EL is found to diverge with the same critical exponent as the correlation length at the QCP. $S(i, j)$ and its first derivative have been found to develop special features in the vicinity of the QCP [7, 18, 19, 20]. The single-site von Neumann entropy (a measure of the entanglement of a single spin with the rest of the system)

$$
S(i)=-\operatorname{Tr} \rho(i) \log _{2} \rho(i)
$$

is also known to be a good indicator of QPTs [3, 18, 19]. In Eq. (3), $\rho(i)$ is the single-site reduced density matrix.

The exploration of the entanglement properties of the ground state of a number of spin-1 Hamiltonians (the AKLT model is an example) has been carried out using both analytical and numerical techniques [11, 12, 13]. Certain spin-1 and generalized spin- $\frac{1}{2}$ ladder model systems are known to have matrix product (MP) states as exact ground states [14, 15, 16]. The MP states are finitely correlated states with short-ranged spin-spin correlations, may have hidden topological order and have gapped excitation spectra. The second order transitions in these so-called finitely correlated MP states belong to the class of generalized QPTs (the definition encompasses the transitions marked by a non-analyticity in any observable of the system) [16] which differ from the conventional QPTs in some important aspects. The spin correlation function in both the cases is of the form $A_{C} e^{-\frac{n}{\xi_{C}}}$ for large $n$. In the case of MP states, $A_{C}$ vanishes at the transition point though the correlation length $\xi_{C}$ blows up as the transition point is approached. In the case of a conventional $\mathrm{QCP}$, the correlation function has a power-law decay close to the QCP. A distinguishing feature of QPTs in MP states arises from the fact that the ground state energy density is analytic for all values of the control parameter. A critical point transition is, however, still signalled by a 
diverging correlation length and the vanishing of an energy gap. The MP states have been used as trial wave functions for a number of standard spin models and provide the basis for the well-known density matrix renormalization group (DMRG) method leading to several interesting developments in quantum information theory [17]. The MP states further serve as candidate systems for the study of unconventional QPTs.

Recently, ground state fidelity has been proposed to provide a signature of QPTs [21, 22] and the usefulness of the measure has been explored in a number of studies [23, 24, 25, 26, 27, 28, 29. Fidelity, a concept borrowed from quantum information theory, is defined as the overlap modulus between ground states corresponding to slightly different Hamiltonian parameters. The advantage of using this measure is that it characterizes QPTs without needing any a priori knowledge of the order parameter and the symmetries of the system. The fidelity typically drops in an abrupt manner at a transition point indicating a dramatic change in the nature of the ground state wave function. A QCP is characterized by the vanishing of the single particle excitation gap. In Ref. 27, an explicit connection between the vanishing of the gap and the fidelity drop has been established. Cozzini et al. 23 tested the validity of the fidelity approach for probing QPTs in MP states and also studied the finite size scaling of the fidelity derivative establishing its relevance in extracting critical exponents. The QPT in the Bose-Hubbard model which is difficult to detect using conventional entanglement measures has been correctly predicted using the fidelity measure [25]. Chen et al. [29] have shown that the fidelity of the first excited state and not the ground state, is the appropriate quantity to signal QPTs in models such as the antiferromagnetic (AFM) Heisenberg spin chain with nearest-neighbour as well as next-nearest-neighbour interactions.

In this paper, we study a $S=\frac{1}{2}$ ladder model with MP states as exact ground states [30]. The model has an interesting phase diagram with two critical point transitions. We explore the properties of the ground state using two different entanglement measures, namely, the single-site and the two-body entanglement. The major motivation is to identify distinctive features, if any, in the entanglement measures close to the QCPs. We look at the same QPTs in the light of fidelity analysis and show that the fidelity $\mathcal{F}$ of the ground state is an efficient indicator of the QPTs. The quantity $\ln |D(u)|$, related to the second derivative of $\mathcal{F}$, also yields useful information regarding the QPTs. We apply the idea of average entanglement [31] to take care of the two-fold degeneracy of the ground state of the model.

\section{ENTANGLEMENT MEASURES}

We consider a general ladder model proposed by Kolezhuk et al. [30] which is described by a Hamiltonian of the general form

$$
\begin{gathered}
H=\sum_{j=1}\left[J\left(S_{1, j} S_{1, j+1}+S_{2, j} S_{2, j+1}\right)+J_{r} S_{1, j} S_{2, j}+V\left(S_{1, j} S_{1, j+1}\right)\left(S_{2, j} S_{2, j+1}\right)\right. \\
+J_{d}\left(S_{1, j} S_{2, j+1}+S_{2, j} S_{1, j+1}\right)+K\left\{\left(S_{1, j} S_{2, j+1}\right)\left(S_{2, j} S_{1, j+1}\right)-\left(S_{1, j} S_{2, j}\right)\left(S_{1, j+1} S_{2, j+1}\right)\right]
\end{gathered}
$$

where the indices 1 and 2 distinguish the lower and upper legs of the ladder and $i$ labels the rungs. The ground state $\left|\psi_{0}(u, \tilde{u})\right\rangle$ has the following MP form

$$
\left|\psi_{0}(u, \tilde{u})\right\rangle=\frac{1}{\sqrt{N_{C}}} \operatorname{Tr}\left\{g_{1}(u) \cdot g_{2}(\tilde{u}) \ldots \ldots g_{2 N-1}(u) \cdot g_{2 N}(\tilde{u})\right)
$$


where

$$
g_{i}(u)=\left(\begin{array}{cc}
u|s\rangle_{i}+\left|t_{0}\right\rangle_{i} & -\sqrt{2}\left|t_{+}\right\rangle_{i} \\
\sqrt{2}\left|t_{-}\right\rangle_{i} & u|s\rangle_{i}-\left|t_{0}\right\rangle_{i}
\end{array}\right)
$$

and $N_{C}\left(=(u \tilde{u}+3)^{2 N}+3(u \tilde{u}-1)^{2 N}\right)$ is the normalization factor. Here $|s\rangle_{i}$ is the singlet state and $\left|t_{\mu}\right\rangle$ with $\mu=+1,0$ and -1 are the triplet states of the $i$-th rung with $S^{z}=+1,0$ and -1 , respectively. $2 N$ is the total number of rungs (with periodic boundary conditions) and $u, \tilde{u}$ are free parameters. For $u \neq \tilde{u},\left|\psi_{0}(u, \tilde{u})\right\rangle$ is dimerized and doubly degenerate as the translation of the rungs by one unit leads to a different state with the same energy.

It is convenient to rewrite the Hamiltonian (5) as a sum of identical local terms which couple only neighbouring rungs, $H=\sum_{j}\left(h_{i, i+1}-E_{0}\right)$. The value of $E_{0}$ is adjusted to make $\left|\psi_{0}\right\rangle$ a zeroenergy ground state which requires the following conditions to be satisfied. (i) All elements of the two matrix products $g_{i}(u) \cdot g_{i+1}(\tilde{u})$ and $g_{i}(\tilde{u}) \cdot g_{i+1}(u)$ have to be zero-energy eigenstates of $h_{i, i+1}$. (ii) The other eigenstates of $h_{i, i+1}$ should have positive energy. The two conditions are satisfied when $h_{i, i+1}$ has the structure

$$
h_{i, i+1}=\sum_{J=0,1,2} \sum_{M=-J}^{J} \epsilon_{J}\left|\psi_{J M}\right\rangle\left\langle\psi_{J M}\right|
$$

where the eigenvalues $\epsilon_{J}>0$ and $\left|\psi_{J M}\right\rangle$ 's are the components of the positive-energy multiplets constructed from the states of the two-rung plaquette $(i, i+1)$ :

$$
\begin{gathered}
\left|\psi_{00}\right\rangle=\left[3+(u \tilde{u})^{2}\right]^{-\frac{1}{2}}\left\{\sqrt{3}|s s\rangle+u \tilde{u}|t t\rangle_{J=0}\right\} \\
\left|\psi_{1}\right\rangle=\left[2+(f)^{2}\right]^{-\frac{1}{2}}\left\{|s t\rangle+|t s\rangle+f|t t\rangle_{J=1}\right\} \\
\left|\psi_{1}\right\rangle=|t t\rangle_{J=2}, \quad f=\frac{u+u}{\sqrt{2}}
\end{gathered}
$$

The notation $|t t\rangle_{J=1}$ has been used to describe states with the total spin $J=1$ constructed from two triplets on rungs $i$ and $i+1$, etc. We obtain the connections between the parameters $J, J_{r}$, $J_{d}, V$ and $K$ of Eq. (4), the local eigenvalues $\epsilon_{J}$ and the singlet weight parameters $u, \tilde{u}$ of the ground state wave function by claiming that the structure (7) is compatible with Eq. (4). The model we study in this paper is a special case of the three types of solutions obtained from the above-mentioned relationships. In this case, $J_{d}=0, K \neq 0$ and

$$
\begin{gathered}
u=-\tilde{u}, K=J_{r}=\epsilon_{0} \frac{\left(u^{2}-1\right)\left(u^{2}+3\right)}{2}, J_{d}=0, \\
V=\epsilon_{0} \frac{\left(5 u^{4}+2 u^{2}+9\right)}{4}, J=3 \epsilon_{0} \frac{\left(u^{4}+10 u^{2}+5\right)}{16}, \\
\epsilon_{1}=\epsilon_{0} \frac{\left(3 u^{4}+14 u^{2}+15\right)}{8}, \epsilon_{2}=\epsilon_{0} \frac{\left(5 u^{4}+18 u^{2}+9\right)}{8}
\end{gathered}
$$

As pointed out in Ref. [30], the one-parameter model undergoes two second-order phase transitions, one at $u=0$ and the other at $u=\infty$. At $u=0$, the ground state undergoes a transition from the dimerized phase to the Haldane phase. The effective Hamiltonian describing this phase is that of the $S=1$ AKLT chain. At $u=\infty$, the transition is to a phase in which the ground state is a product of singlet bonds on the rungs. The transitions at $u=0$ and $\infty$ are marked by the vanishing of the singlet and triplet gaps, respectively, in the excitation spectrum [30]. The ground state is spontaneously dimerized everywhere except at the critical points. In the MP formalism, it is straightforward to calculate the spin-spin and dimer-dimer correlation functions $C_{S}(n)=\left\langle S_{1, i}^{z} S_{1, i+n}^{z}\right\rangle$ and $C_{D}(n)=\left\langle D_{i} D_{i+n}\right\rangle$ where $D_{i}=\mathbf{S}_{1, i} \cdot\left(\mathbf{S}_{1, i+1}-\mathbf{S}_{1, i-1}\right)$. The dimer correlations are long-ranged and vanish as $u \rightarrow 0, \infty$ but with no exponential tail. The spin correlation 
length is finite at the AKLT point $u=0$, becomes zero at $u=1$ and diverges as $u \rightarrow \infty$. There is, however, no development of long-range spin order since the amplitude of the spin correlations becomes zero in this limit. The doubly-degenerate spontaneously dimerized phase which prevails away from the critical points exhibits non-Haldane spin liquid properties. The elementary excitation is of a novel type, a pair of propagating triplet or singlet solitons connecting two spontaneously dimerized ground states [30]. In the Haldane phase, the elementary excitation has the character of a magnon.

Using the transfer matrix (TM) method, we now study the entanglement properties of the MP ground state [Eq. (9)]. The state is two-fold degenerate as the ground-state energy per rung $E_{0}=-\frac{3}{64} \lambda_{0}\left(7 u^{4}+22 u^{2}+19\right)$ does not depend on the sign of $u$. The two ground states obtained from Eq. (6)

$$
\begin{aligned}
& \left|\psi_{1}\right\rangle=\frac{1}{\sqrt{N_{0}(u)}} \operatorname{Tr}\left\{g_{1}(u) \cdot g_{2}(-u) \ldots \ldots . g_{2 N-1}(u) \cdot g_{2 N}(-u)\right\} \\
& \left|\psi_{2}\right\rangle=\frac{1}{\sqrt{N_{0}(u)}} \operatorname{Tr}\left\{g_{1}(-u) \cdot g_{2}(u) \ldots \ldots . g_{2 N-1}(-u) \cdot g_{2 N}(u)\right\}
\end{aligned}
$$

are asymptotically orthogonal in the thermodynamic limit (TDL) $N \rightarrow \infty$, i.e., the overlap $\left\langle\psi_{1} \mid \psi_{2}\right\rangle=\frac{3\left(u^{2}+1\right)^{2 N}+\left(u^{2}-3\right)^{2 N}}{\left(u^{2}+3\right)^{2 N}+3\left(u^{2}-1\right)^{2 N}} \leq 1$ for finite $N$ and vanishes in the limit $N \rightarrow \infty$. $N_{0}(u)[=$ $\left.\left(u^{2}+3\right)^{2 N}+3\left(u^{2}-1\right)^{2 N}\right]$ is the normalization factor. We construct a pair of orthogonal degenerate ground states applying the usual Gram-Schmidt procedure

$$
\begin{gathered}
\left|\phi_{1}\right\rangle=\left|\psi_{1}\right\rangle \\
\left|\phi_{2}\right\rangle=\frac{1}{\sqrt{\widetilde{N}}}\left(\left|\psi_{2}\right\rangle-\left\langle\psi_{1} \mid \psi_{2}\right\rangle\left|\psi_{1}\right\rangle\right)
\end{gathered}
$$

with $\tilde{N}=1-\left|\left\langle\psi_{1} \mid \psi_{2}\right\rangle\right|^{2}$. An arbitrary superposition of the two degenerate ground states is also a valid ground state. We apply the idea of average entanglement [31], i.e., calculate the entanglement content of a general state (an arbitrary superposition of basis states) and then calculate its average value over the whole of parameter space (the coefficients of the basis-state expansion constitute the parameters)

$$
E_{a v}=\frac{\int d \mu\left(p_{1}, p_{2}, \ldots\right)\left|E\left(p_{1}, p_{2}, \ldots\right)\right|}{\int d \mu\left(p_{1}, p_{2}, \ldots\right)}
$$

where $\int d \mu\left(p_{1}, p_{2}, \ldots\right)$ is the Haar measure associated with the parametrization $p_{1}, p_{2}, \ldots$, which is invariant under unitary operations. The normalization condition restricts the values of the coefficients so that the parameter space is associated with a compact hyper-surface. In the case of a double degenerate ground state, a general state is a superposition of two states

$$
\left|\phi_{s}\right\rangle=a\left|\phi_{1}\right\rangle+b\left|\phi_{2}\right\rangle
$$

with the restriction $|a|^{2}+|b|^{2}=1$. The corresponding parameter space is a $3-D$ sphere $S^{3}$. The one-rung reduced density matrix $\rho(i)$ (Eq. (3)) is obtained by tracing out all the rungs except the $i$-th one from the ground state density matrix $\rho=\left|\phi_{s}\right\rangle\left\langle\phi_{s}\right|$. From Eq. (13)

$$
\rho(i)=\operatorname{Tr}_{1, . . L}^{i}\left|\phi_{s}\right\rangle\left\langle\phi_{s}\right|=\operatorname{Tr}_{1, . . L}^{i}\left(|a|^{2}\left|\phi_{1}\right\rangle\left\langle\left.\phi_{1}|+| b\right|^{2} \mid \phi_{2}\right\rangle\left\langle\phi_{2}\left|+a b^{*}\right| \phi_{1}\right\rangle\left\langle\phi_{2}\left|+a^{*} b\right| \phi_{2}\right\rangle\left\langle\phi_{1}\right|\right.
$$

With the help of standard TM calculations [19], one obtains a form for $\rho(i)$ which is found to be 
independent of the parameters $a$ and $b$ in the TDL,

$$
\rho(i)=\left(\begin{array}{cccc}
\frac{1}{u^{2}+3} & 0 & 0 & 0 \\
0 & \frac{1}{u^{2}+3} & 0 & 0 \\
0 & 0 & \frac{1}{u^{2}+3} & 0 \\
0 & 0 & 0 & \frac{u^{2}}{u^{2}+3}
\end{array}\right)
$$

in the $\left|t_{ \pm 1,0}, s\right\rangle$ basis. The single-rung entanglement is obtained as

$$
S(i)=\frac{1}{u^{2}+3}\left[\left(u^{2}+3\right) \log _{2}\left(u^{2}+3\right)-u^{2} \log _{2} u^{2}\right]
$$

Entanglement average, as defined in Eq. (12), is required for finite-sized systems. In the TDL, such averaging is not necessary as $\rho(i)$ [Eq. (14)] is independent of $a$ and $b\left(|a|^{2}+|b|^{2}=1\right)$. The variations of $S(i)$ and its first derivative with respect to $u$ have been shown in Fig. 1 (top) and (bottom) respectively. $S(i)$ has the value $\log _{2} 3$ at the critical point $u=0$ (the AKLT point) as expected, increases as $u$ is increased from zero before it reaches its maximum possible value of 2 at $u=1$. Then it decreases with increasing $u$ and vanishes at the other transition point $u=\infty$ (Fig. 2 ). In the rung-singlet phase, each pair of spins in a rung forms a singlet to become maximally entangled with each other and completely unentangled with the rest of the system. The plots are expectedly symmetric about the point $u=0$.

The two-rung reduced density matrix $\rho(i, j)$ can be calculated in the same manner. $\rho(i, j)$ is given by

$$
\rho(i, j)=\operatorname{Tr}_{1, . . L}^{i, j}\left|\phi_{s}\right\rangle\left\langle\phi_{s}\right|
$$

where the trace is taken over all the rungs except the $i$-th and $j$-th ones. From the usual TM calculations, we obtain $\rho(i, j)$, in the TDL, as a $16 \times 16$ matrix in block-diagonal form. From (1) and (17), the two-body entanglement is

$$
S(i, j)=-\sum_{i} \lambda_{i} \log _{2} \lambda_{i}
$$

$\lambda_{i}$ 's being the eigenvalues of $\rho(i, j)$. Figure 3 shows the variation of the average $S(i, j)$ (top) and its first derivative (bottom) with $u$ for $n=1000 . S(i, j)$ behaves in a similar manner as $S(i)$. It has the value $2 \log _{2} 3$ at the QCP $u=0$, it then increases with $u$ to attain the peak value 4 at $u=1$ and when $u$ is increased further, $S(i, j)$ decreases and falls to zero (Fig. 4) as we approach the QCP $u=\infty$. The first derivatives of $S(i)$ and $S(i, j)$, instead of showing any non-analyticity, fall sharply to zero at both the QCPs. The first derivatives are also zero at $u=1$ where the entanglement measures have the maximum value. The second derivatives of $S(i)$ and $S(i, j)$ are logarithmically divergent at both the QCPs $u=0$ and $\infty$ (as can be seen in the insets of Figs. $1-4$ ). Both the measures $S(i)$ and $S(i, j)$ vanish at $u=\infty$ and are non-zero elsewhere, they thus behave as an order parameter decreasing to zero value at the QCP $u=\infty$ with transition to the rung-singlet phase. The measures, however, do not have the character of an order parameter for the transition at $u=0$ from the dimerized to the Haldane phase.

We next calculate the EL, $\xi_{E}$, associated with the entanglement measure $S(i, j)$. Close to either of the QCPs and in the limit of large $n$, we have $S(n=|j-i|)-S(\infty) \sim A_{e} e^{-\frac{n}{\xi_{E}}}$. The pre-factor $A_{e}$ is found to remain finite and non-zero at the transition point $u=0$ but it vanishes at $u=\infty$. The EL $\xi_{E}$ is given by

$$
\xi_{E}=\frac{1}{2 \ln \left|\frac{u^{2}+3}{u^{2}-1}\right|}
$$


We rewrite $\xi_{E}$ as a function of $\frac{1}{u}$, i.e., $\xi_{E}=\frac{1}{2 \ln \left|\frac{1+3\left(\frac{1}{u}\right)^{2}}{1-\left(\frac{1}{u}\right)^{2}}\right|}$ and study its behaviour near $u=\infty$, i.e., $\frac{1}{u}=0$. Fig. 5 shows the variation of $\xi_{E}$ with respect to $u$ and $\frac{1}{u}$. We find that $\xi_{E}$ is finite at the critical point $u=0$ but it diverges as $u \rightarrow \infty$ with the critical exponent $\nu=2$ as $\xi_{E} \sim\left(\frac{1}{u}\right)^{-2}$ for $\frac{1}{u} \sim 0$. The spin-spin correlation function $C_{S}(n)=<S_{1, i}^{z} S_{1, i+n}^{z}>$ can be calculated in the TM formalism as [30]

$$
\begin{gathered}
C_{S}(n)=\left(u^{2}+3\right)^{-1}\left(z_{+} z_{-}\right)^{n}\left(\delta_{n, 2 k}-z_{-} \delta_{n, 2 k+1}\right) \\
z_{ \pm}=(u \pm 1)^{2} /\left(u^{2}+3\right)
\end{gathered}
$$

Close to the QCP $u=\infty, \xi_{E} \sim \xi_{C} / 2$ so that both $\xi_{E}$ and $\xi_{C}$ diverge with the same exponent $\nu=2$.

\section{GROUND STATE FIDELITY $\mathcal{F}(u, \delta)$}

We now investigate the behaviour of fidelity near the same pair of QCPs. The average fidelity, in analogy to $(12)$, is

$$
\mathcal{F}_{a v}=\frac{\int d \mu\left(p_{1}, p_{2}, \ldots\right) \mathcal{F}\left(p_{1}, p_{2}, \ldots\right)}{d \mu\left(p_{1}, p_{2}, \ldots\right)}
$$

The overlap between two general ground states, $\left|\phi\left(u_{1}\right)\right\rangle$ and $\left|\phi\left(u_{2}\right)\right\rangle$ (see Eq.(13)), corresponding to two different values of the control parameter is given by

$$
\mathcal{F}\left(u_{1}, u_{2}\right)=\left\langle\phi\left(u_{1}\right) \mid \phi\left(u_{2}\right)\right\rangle=|a|^{2}\left\langle\phi_{1} \mid \phi_{1}\right\rangle+|b|^{2}\left\langle\phi_{2} \mid \phi_{2}\right\rangle+a b^{*}\left\langle\phi_{1} \mid \phi_{2}\right\rangle+a^{*} b\left\langle\phi_{2} \mid \phi_{1}\right\rangle
$$

$\mathcal{F}\left(u_{1}, u_{2}\right)$ (averaged over the $\{a, b\}$ ) can be expressed in terms of the eigenvalues of the TM [23] as

$$
\begin{gathered}
\mathcal{F}\left(u_{1}, u_{2}\right)=\frac{1}{\sqrt{N_{0}\left(u_{1}\right) N_{0}\left(u_{2}\right)}}\left[( 1 + \frac { 1 + p ( u _ { 1 } ) p ( u _ { 2 } ) } { \sqrt { ( 1 - p ^ { 2 } ( u _ { 1 } ) p ^ { 2 } ( u _ { 2 } ) ) } } ) \left\{\left(u_{1} u_{2}+3\right)^{2 N}+3\right.\right. \\
\left.\left.\left(u_{1} u_{2}-1\right)^{2 N}\right\}-\frac{p\left(u_{1}\right) p\left(u_{2}\right)}{\sqrt{\left(1-p^{2}\left(u_{1}\right) p^{2}\left(u_{2}\right)\right)}}\left\{\left(u_{1} u_{2}-3\right)^{2 N}+3\left(u_{1} u_{2}+1\right)^{2 N}\right\}\right]
\end{gathered}
$$

where $p(u)=\frac{3\left(u^{2}+1\right)^{2 N}+\left(u^{2}-3\right)^{2 N}}{\left(u^{2}+3\right)^{2 N}+3\left(u^{2}-1\right)^{2 N}}$. Fig. $6($ top $)$ shows the nature of the variation of $\mathcal{F}(u, u+\delta)$, (overlap of the states corresponding to two close points in the control parameter space separated by a small variation) with $u$ and $N$ in the neighbourhood of the critical point $u=0$ for $\delta=$ .001. A straightforward calculation reveals that for large values of $N$ and for $u \neq 0, \mathcal{F}\left(u_{1}, u_{2}\right) \sim$ $\left(\alpha\left(u_{1}, u_{2}\right)\right)^{N}$, where $\alpha\left(u_{1}, u_{2}\right)=\frac{u_{1}^{2} u_{2}^{2}+6 u_{1} u_{2}+9}{u_{1}^{2} u_{2}^{2}+3\left(u_{1}^{2}+u_{2}^{2}\right)+9} \cdot \alpha(u, u+\delta)<1$ and it has a sharp dip at $u=0$. Thus away from the critical point, $\mathcal{F}(u, u+\delta)$ decreases exponentially with $N$ and vanishes in the TDL for any fixed value of $u$ and $\delta$, but we observe from Fig. 6 (top) that $\mathcal{F}(u, u+\delta)$ decreases at a much enhanced rate when the QCP is approached. Intuitively, the rate of orthogonality, i.e., the rate at which the "distance" between the ground states corresponding to two neighbouring points of the parameter space becomes maximal, should diverge in the proximity of a QPT. It is thus sensible to relate the degree of criticality to the derivative of the fidelity function. Cozzini et al [23] have proposed a general expression for the quantity relevant in this case

$$
D(u)=-\left.\partial_{u_{1}} \partial_{u_{2}} \ln F\left(u_{1}, u_{2}\right)\right|_{u_{1}=u_{2}=u}
$$

where $F\left(u_{1}, u_{2}\right)=\sqrt{N_{0}\left(u_{1}\right) N_{0}\left(u_{2}\right)} \mathcal{F}\left(u_{1}, u_{2}\right)$. In the large $N$ limit and for $u \neq 0$, one can easily check that $D(u) \sim \frac{N}{\left(u^{2}+3\right)^{2}}$. Thus in the plots (Fig. 6 (bottom)) showing the variation of $\ln |D(u)|$ 
with $u$ for different values of $N$, we observe that the rate at which $\ln |D(u)|$ increases with $u$ is heightened in the proximity of the QCP $u=0$. To repeat the whole analysis for the other critical point $u=\infty$, we express $F\left(u_{1}, u_{2}\right)$ as a function of $\tilde{u_{1}}=\frac{1}{u_{1}}$ and $\tilde{u_{2}}=\frac{1}{u_{2}}$. For very large $N$, $\mathcal{F}^{\prime}\left(\tilde{u}_{1}, \tilde{u}_{2}\right) \sim\left(\alpha^{\prime}\left(u_{1}, u_{2}\right)\right)^{N}\left[\alpha^{\prime}\left(\tilde{u}_{1}, \tilde{u}_{2}\right)=\frac{9 \tilde{u}_{1}^{2} \tilde{u}_{2}^{2}+6 \tilde{u}_{1} \tilde{u}_{2}+1}{9 \tilde{u}_{1}^{2} \tilde{u}_{2}^{2}+3\left(\tilde{u}_{1}^{2}+\tilde{u}_{2}^{2}\right)+1}\right]$ and $D^{\prime}(\tilde{u}) \sim \frac{N}{\left(3 \tilde{u}^{2}+1\right)^{2}}$ away from the critical point. We find a similar variation of $\mathcal{F}^{\prime}(\tilde{u}, \tilde{u}+\delta)$ [Fig. 7 (top)] and $D^{\prime}(\tilde{u})$ [Fig. 7 (bottom) ] near the QCP $\tilde{u}=0$, i.e., $u=\infty$ as in the case of the QCP $u=0 . \mathcal{F}^{\prime}(\tilde{u}, \tilde{u}+\delta)$ falls sharply at $\tilde{u}=0$ and the fall becomes faster as we increase $N$. The quantity $l n\left|D^{\prime}(\tilde{u})\right|$ increases at an enhanced rate and tends to blow up in the vicinity of $\tilde{u}=0$ as we increase the value of $N$. The inset of the figure shows that curves plotted in rescaled units collapse onto a single curve for different values of $N$. The rescaled quantity $\frac{D^{\prime}(\tilde{u})}{N}$ is found to be a function of $N \tilde{u}^{2}$ only. This feature of data collapse is analogous to the scaling behaviour of observables in the vicinity of a critical point. The finite size scaling hypothesis, valid in the critical region, is given by $X_{N}=N^{\frac{\rho}{\nu}} Q\left(N\left|g-g_{c}\right|^{\nu}\right)$ where $X_{N}$ is some observable with the divergent behaviour $X_{N} \sim\left|g-g_{c}\right|^{-\rho}$ close to the critical point $g=g_{c}$. The exponent $\nu$ is the correlation length exponent. In the present case $\rho \sim 0$ and $\nu=2$.

\section{DISCUSSIONS}

In this paper, we have studied a $S=\frac{1}{2}$ spin ladder model the exact ground states of which are MP states. The ground state is spontaneously dimerized and doubly degenerate (broken translational symmetry) at all values of the parameter $u$ excepting the points at $u=0$ and $u=\infty$. At $u=0$, a QPT occurs to the Haldane phase of an effective $S=1$ chain which is signalled by the vanishing of a singlet excitation gap. The elementary singlet excitation in the dimerized phase is neither a magnon nor a spinon but a soliton in the dimer order. The lowest soliton excitations occur in pairs. At $u=\infty$, there is another QPT to the rung-singlet phase with the vanishing of a triplet excitation gap, associated with triplet solitons. The ground states in all the three phases: Haldane $(u=0)$, spontaneously dimerized $(0<u<\infty)$ and rung-singlet $(u=\infty)$ are spin liquids with no conventional long-range order in the two-spin correlation functions but are characterized by other types of order parameters. The spontaneously dimerized phase has long range order in dimer correlations which vanishes for $u \rightarrow 0, \infty$ but there is no exponential tail. The Haldane phase has the string order parameter [11, 12, 13, 14] whereas the rung-singlet phase has dimer-dimer correlations with the dimers located on the rungs. The two-spin correlation length is finite at $u=0$ and diverges as $u \rightarrow \infty$ but no long range order develops in the latter case since the amplitude of spin correlations falls to zero in this limit.

As pointed out in [16, QPTs in MP states are unconventional with the ground state energy analytic at $g=g_{c}$, the transition point. A conventional QPT is signalled by a non-analyticity in the ground state energy. One can, however, generalize the definition of QPT to include cases where any observable quantity becomes non-analytic as the transition point is reached. MP states are an important class of states which provide an exact representation of many-body ground states of specific Hamiltonians. Also, every state of a finite system has an MP representation which thus provides the basis of the powerful DMRG method. In the thermodynamic limit, second order QPTs occur in MP ground states accompanied by vanishing energy gaps and diverging correlation lengths. We have studied the variation of the entanglement measures $S(i)$ and $S(i, j)$ as a function of $u$ in the ground state of the spin ladder model with QCPs at $u=0$ and $\infty$. The major goal of our study is to identify signatures of QPTs, if any, in the quantum information theoretic measures associated 
with entanglement and fidelity. We provide a summary and analysis of our results below.

Both $S(i)$ and $S(i, j)$ have zero values at $u=\infty$, i.e., in the rung singlet phase (Figs. 2 and 4) and nonzero values in the dimerized phase $0<u<\infty$. The entanglement measures can thus be treated as an order parameter with zero value at the QCP $u=\infty$ and non-zero value in the preceding dimerized phase. In the rung singlet phase, each rung is described by a spin singlet which is maximally entangled but the rung is disentangled from the rest of the system. The EL, $\xi_{E}$, as calculated from $S(i, j)$ diverges as $u \rightarrow \infty$ (Fig. 5 (bottom)) with $\xi_{E}=\frac{\xi_{C}}{2}, \xi_{C}$ being the spin-spin correlation length. The entanglement content in this case vanishes with infinite entanglement range. At the QCP $u=0$, the entanglement measures have the magnitudes associated with the AKLT state of a spin-1 model. The entanglement measure has a local minimum at this point, rises to the maximum value at $u=1$ and then decreases to the global minimum value zero at $u=\infty$. The first derivatives of $S(i)$ and $S(i, j)$ both fall sharply to zero at $u=0$ and $u=\infty$. The double derivatives of these quantities diverge as the QCPs are approached (insets of Figs. 1-4). The divergence arises from the structure of the von Neumann entropy involving terms such as $\log _{2} u^{2}$ or $\log _{2} \frac{1}{u^{2}}$. A similar type of divergence occurs in the QPT of a model studied in [6]. We thus find that the entanglement measures $S(i)$ and $S(i, j)$ do develop distinctive features close to the QCPs $u=0$ and $u=\infty$.

We further looked for signatures of QPTs via the fidelity measure. Fidelity, i.e., the overlap of ground states for slightly different Hamiltonian parameters, is expected to drop abruptly at a QCP indicating a dramatic change in the ground state structure. We plotted $\mathcal{F}(u, \delta)=\langle u \mid u+\delta\rangle$ with $u$ and $N$ for $\delta=10^{-3}$ and found that the quantity indeed falls to zero rapidly as the QCPs $u=0$ and $\infty$ are approached. The quantity $\ln |D(u)|$, where $D(u)$ is related to the second derivative of $F$, also provides a good signature of QPTs. You et al [32] has introduced a quantity, the so-called fidelity susceptibility $\chi_{F}$ which is defined as

$$
\chi_{F}(u)=\lim _{\delta \rightarrow 0} \frac{-2 \ln \mathcal{F}(u, \delta)}{\delta^{2}}
$$

One can easily check that $\chi_{F}$ has the same form as $D(u)$. The finite size scaling hypothesis, which is expected to be valid in the vicinity of a $\mathrm{QCP}$, leads to the collapse of curves onto a single scaling function (inset of Fig. 7) as the QCP $u=\infty$ is approached. The fidelity measures exhibit similar features in the case of a conventional QPT. The spin ladder model studied in the paper has spin liquid-type ground states with none of the phases exhibiting long range magnetic order in the twospin correlation functions. The model has three distinct phases with characteristic quantum order parameters. A characterization of the transitions between the phases in terms of entanglement and fidelity measures provide a new perspective on the many body finitely correlated states and the transitions between them. Quantum information theoretic measures such as entanglement and

fidelity provide a novel characterization of QPTs occuring in many-body condensed matter systems [33, 34, 35]. The present study illustrates this in the case of a spin ladder model with spin-liquid type ground states.

\section{ACKNOWLEDGMENT}

A. T. is supported by the Council of Scientific and Industrial Research, India, under Grant No. 9/15 (306)/ 2004-EMR-I. 


\section{References}

[1] S. Sachdev, Quantum Phase Transitions (Cambridge University Press, Cambridge, 2000); S. L. Sondhi et al., Rev. Mod. Phys. 69, 315 (1997).

[2] A. Osterloh, L. Amico, G. Falci and R. Fazio, Nature 416, 608 (2002).

[3] T. J. Osborne and M. A. Nielsen, Phys. Rev. A 66, 032110 (2002).

[4] G. Vidal, J. I. Latorre, E. Rico and A. Kitaev, Phys. Rev. Lett. 90, 0279011 (2004).

[5] L. -A. Wu, M. S. Sarandy and D. A. Lidar, Phys. Rev. Lett 93, 250404 (2004).

[6] A. Tribedi and I. Bose, Phys. Rev. A 75, 042304 (2007).

[7] H.- D. Chen, J. Phys. A: Math. Theor. 40, 10215 (2007).

[8] I. Bose and E. Chattopadhyay, Phys. Rev. A 66, 062320 (2002).

[9] F. C. Alcaraz, A. Saguia and M. S. Sarandy, Phys. Rev. A 70, 032333 (2004).

[10] J. Vidal, R. Mosseri and J. Dukelsky, Phys. Rev. A 69, 054101 (2004).

[11] F. Verstraete, M. Popp and J. I. Cirac, Phys. Rev. Lett. 92, 027901 (2004).

[12] H. Fan, V. Korepin and V. Roychowdhury, Phys. Rev. Lett. 93, 227203 (2004).

[13] F. Verstraete, M. A. Martin-Delgado and J. I. Cirac, Phys. Rev. Lett. 92, 087201 (2004).

[14] A. Klümper, A. Schadschneider and J. Zittartz, Z. Phys. B 87, 281-287 (1992).

[15] A. K. Kolezhuk and H. J. Mikeska, Int. J. Mod. Phys. B 12, 2325 (1998) and references therein.

[16] M. Fannes, B. Nachtergaele and R. F. Werner, Europhys. Lett. 10, 633 (1989); Commun. Math. Phys. 144, 443 (1992).

[17] M. M. Wolf, G. Ortiz, F. Verstraete and J. I. Cirac, Phys. Rev. Lett. 97, 110403 (2006).

[18] D. Pérez-García, F. Verstraete, M. M. Wolf and J. I. Cirac, Quantum Inf. Comput. 7, 401 (2007).

[19] M. Asoudeh, V. Karimipour and A. Sadrolashrafi, Phys. Rev. B 75, 224427 (2007).

[20] S. Alipour, V. Karimipour, and L. Memarzadeh, Phys. Rev. A 75, 052322 (2007).

[21] H. T. Quan, Z. Song, X. F. Liu, P. Zanardi and C. P. Sun, Phys. Rev. Lett. 96, 140604 (2006).

[22] P. Zanardi and N. Paunkovic, Phys. Rev. E 74, 031123 (2006).

[23] M. Cozzini, R. Ionicioiu and P. Zanardi, Phys. Rev. B 76, 104420 (2007).

[24] M. Cozzini, P. Giorda and P. Zanardi, Phys. Rev. B 75, 014439 (2007).

[25] P. Buonsante and A. Vezzani, Phys. Rev. Lett. 98, 110601 (2007). 
[26] H. -Q. Zhou, e-print arXiv:0704.2945.

[27] P. Zanardi, M. Cozzini and P. Giorda, J. Stat. Mech.: Theory Exp. (2007), L02002.

[28] P. Zanardi, H. T. Quan, X. Wang and C. P. Sun, Phys. Rev. A 75, 032109 (2007).

[29] S. Chen, L. Wang, S. J. Gu and Y. Wang, Phys. Rev. E 76, 061108 (2007).

[30] A. K. Kolezhuk and H. J. Mikeska, Phys. Rev. Lett. 80, 2709 (1998)

[31] Y. -Q. Li, G. -Q. Zhu and X. -A. Zhao, e-print arXiv:quant-ph/0408155.

[32] W. L. You, Y. W. Li and S. J. Gu, Phys. Rev. E 76, 022101 (2007).

[33] L. Amico, R. Fazio, A. Osterloh and V. Vedral, e-print arXiv:quant-ph/0703044.

[34] M. Lewenstein, A. Sanpera, V. Ahufinger, B. Damski, A. Sen(de) and U. Sen, Adv. Phys. 56, 243 (2007).

[35] L. Campos Venuti, M. Cozzini, P. Buonsante, F. Massel, N. Bray-Ali and P. Zanardi, e-print arXiv: 0801.2473 . 

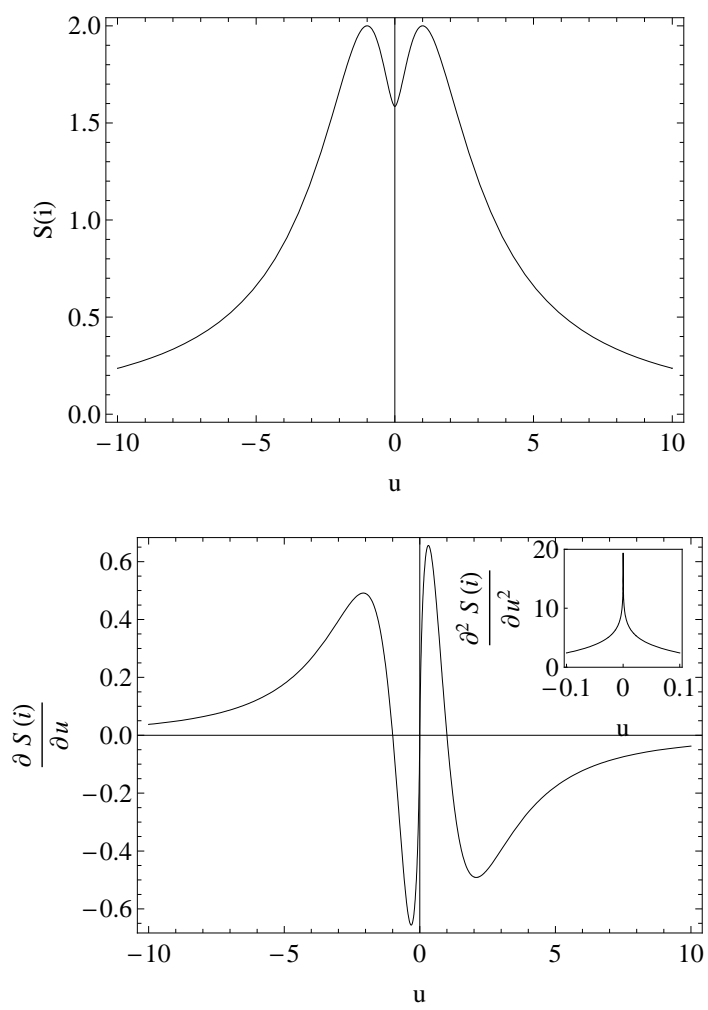

FIG. 1: Plot of $S(i)$ (top) and $\frac{\partial S(i)}{\partial u}$ (bottom) as functions of $u$. The inset (bottom) shows the diverging behavior of the second derivative of $S(i)$ near $u=0$. 

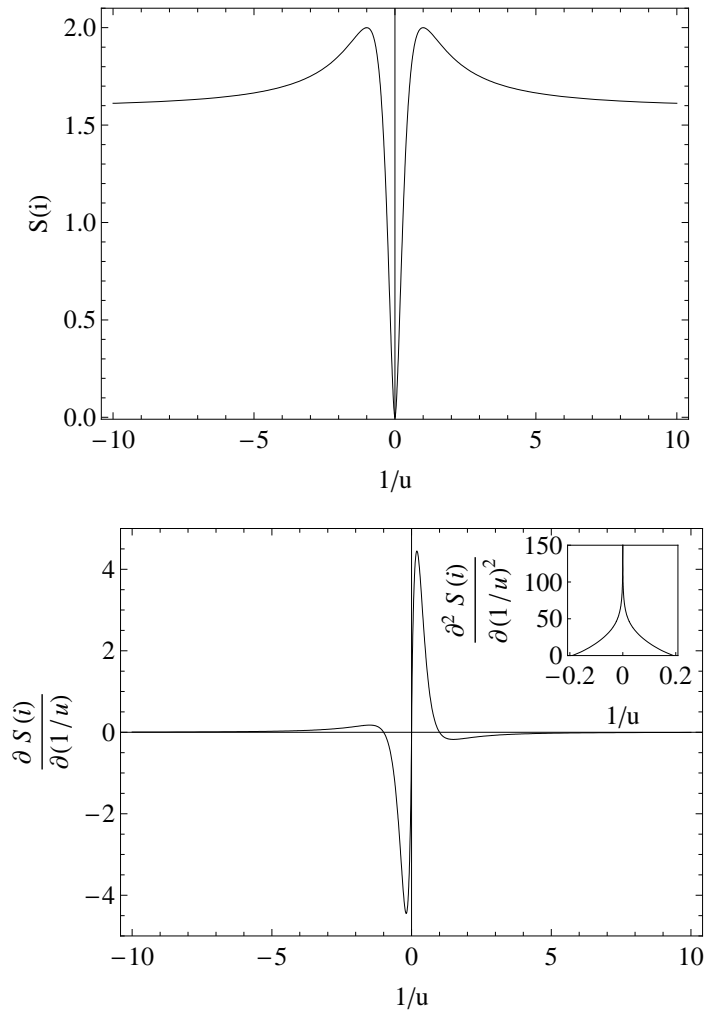

FIG. 2: Plot of $S(i)$ (top) and $\frac{\partial S(i)}{\partial\left(\frac{1}{u}\right)}$ (bottom) as functions of $\frac{1}{u}$. The inset (bottom) shows the diverging behavior of the second derivative of $S(i)$ near $u=\infty$. 

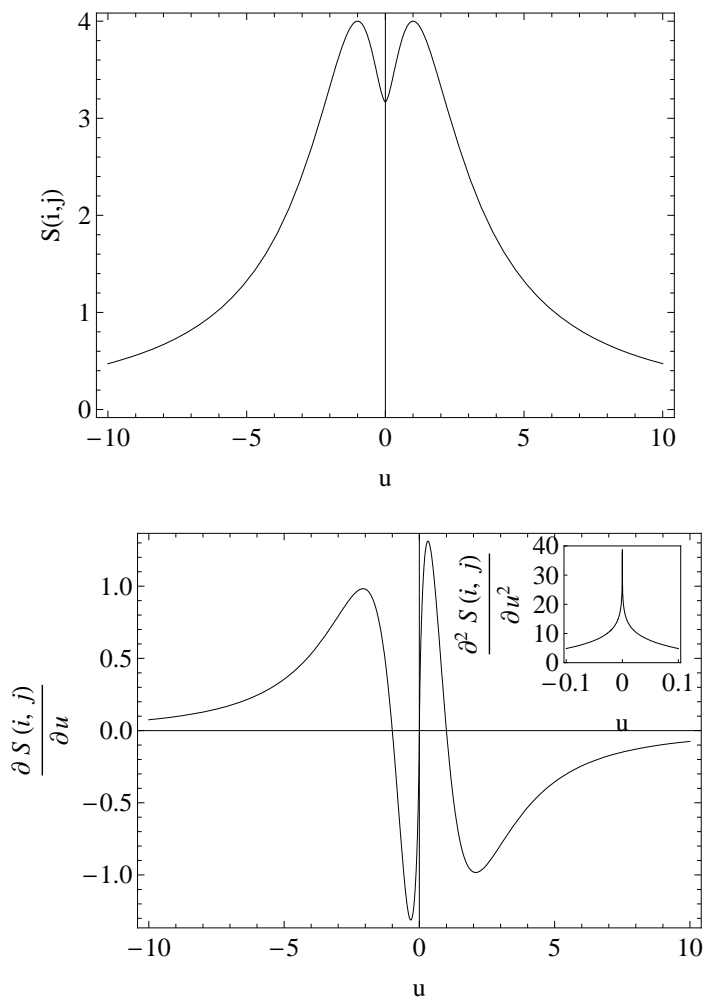

FIG. 3: Plot of $S(i, j)$ (top) and $\frac{\partial S(i, j)}{\partial u}$ (bottom) as functions of $u$ for $n=1000$. The inset (bottom) shows the diverging behavior of the second derivative of $S(i, j)$ near $u=0$. 

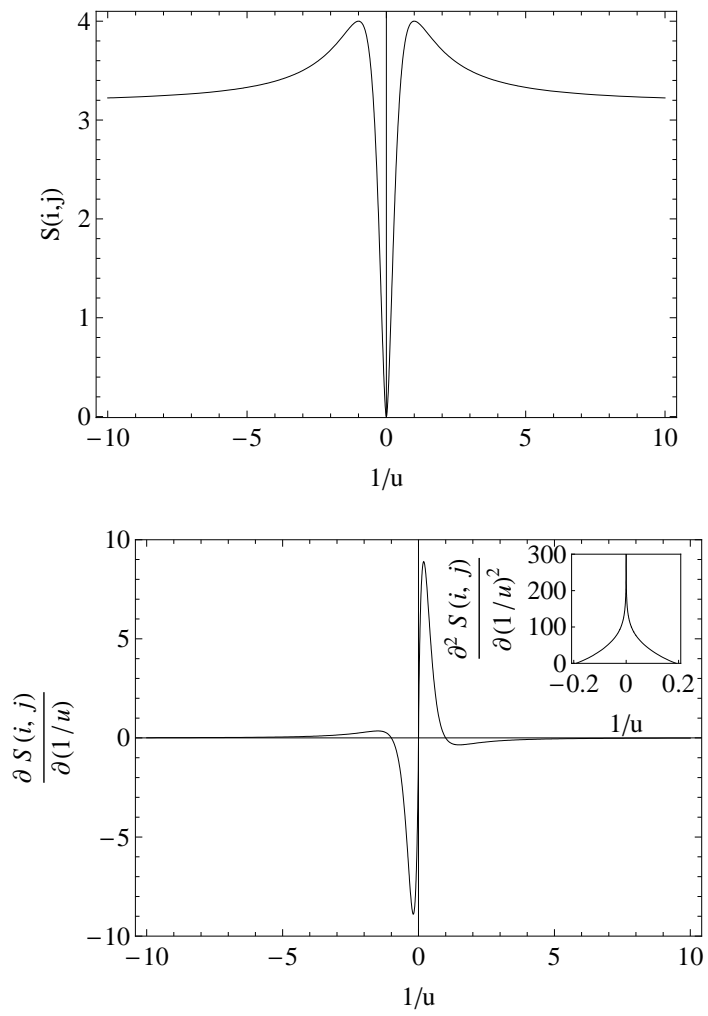

FIG. 4: Plot of $S(i, j)$ (top) and $\frac{\partial S(i, j)}{\partial\left(\frac{1}{u}\right)}$ (bottom) as functions of $\frac{1}{u}$ for $n=1000$. The inset (bottom) shows the diverging behavior of the second derivative of $S(i, j)$ near $u=\infty$. 

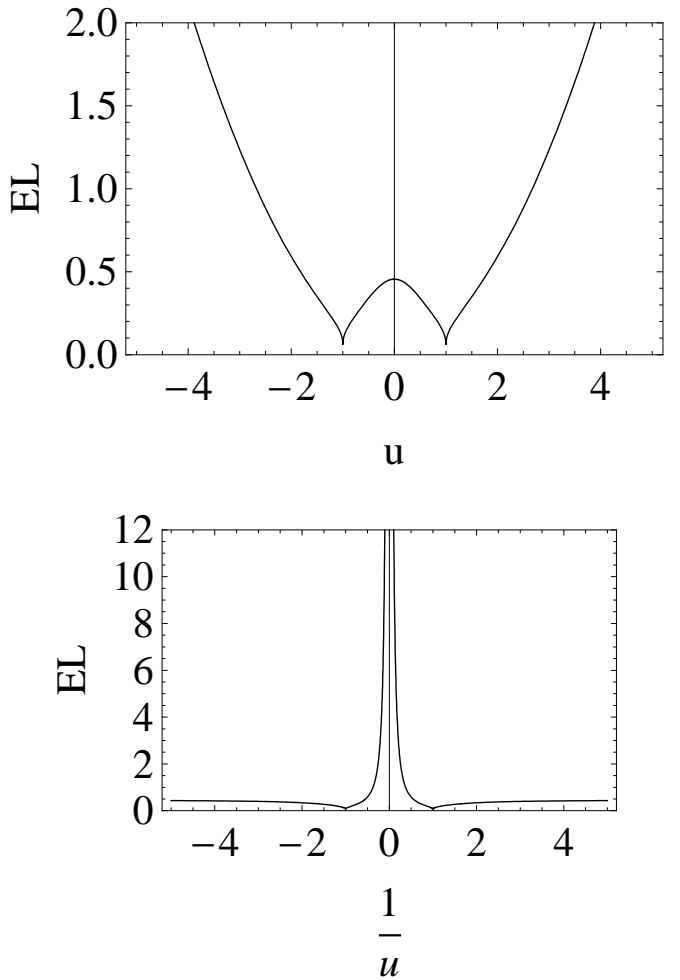

FIG. 5: Plot of EL as a function of $u$ (top) and $\frac{1}{u}$ (bottom) . 

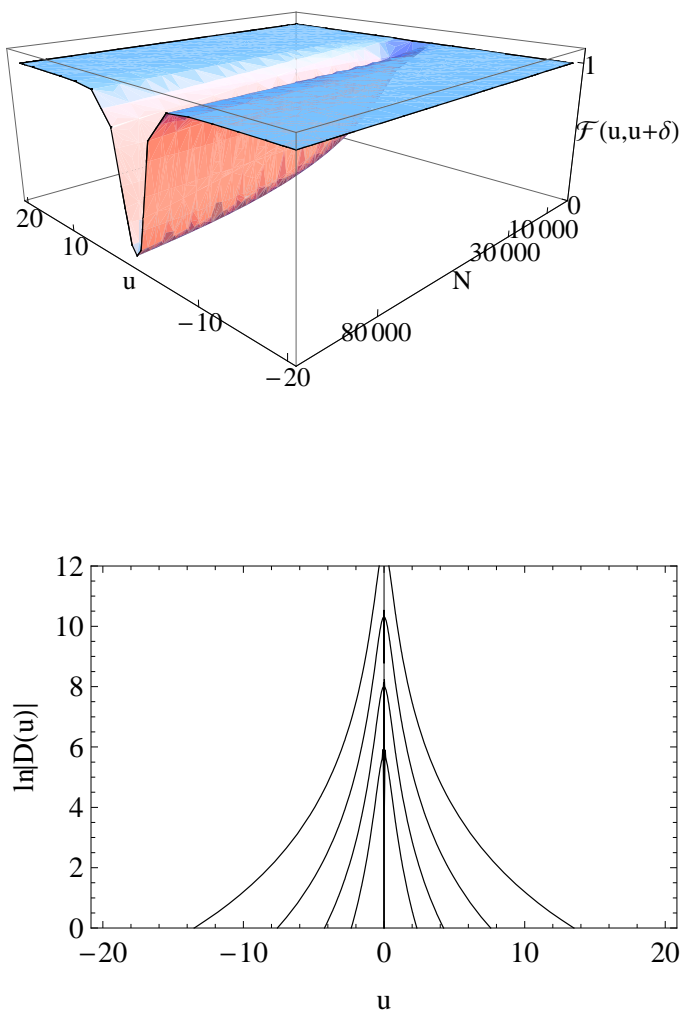

FIG. 6: Plot of $\mathcal{F}(u, u+\delta)$ (top) as a function of $u$ and $N$ for $\delta=.001$ and $\ln |D(u)|$ (bottom) $\left(N=10^{3}, 10^{4}, 10^{5}\right.$ and $\left.10^{6}\right)$ as a function of $u$. 

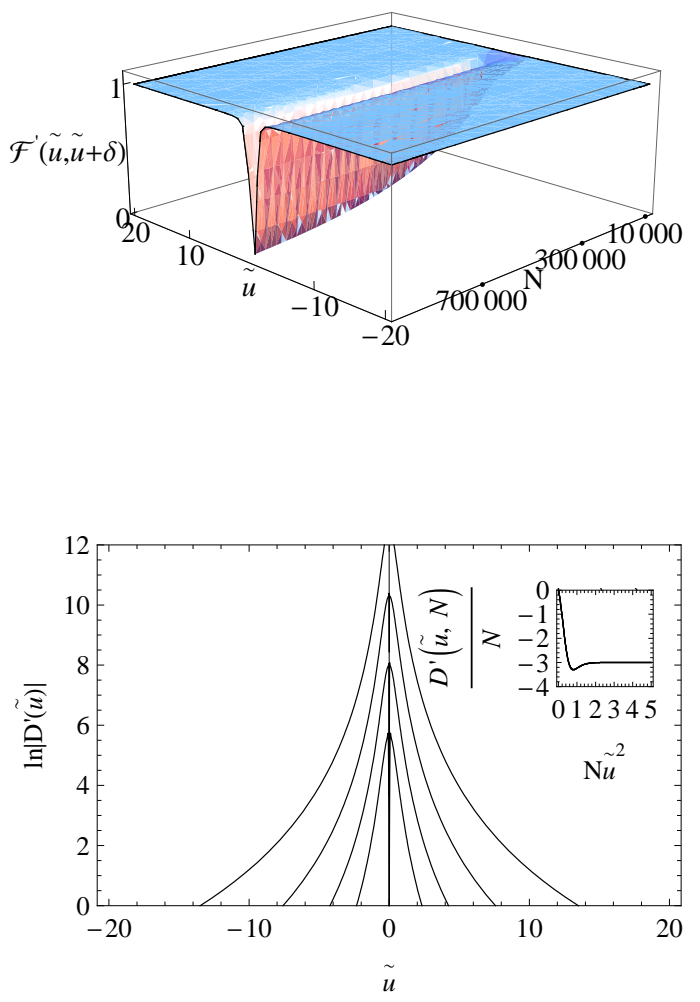

FIG. 7: Plot of $\mathcal{F}^{\prime}(\tilde{u}, \tilde{u}+\delta)$ (top) as a function of $\tilde{u}$ and $N$ for $\delta=.001$ and $\ln \left|D^{\prime}(\tilde{u})\right|$ (bottom) $\left(N=10^{2}, 10^{3}, 10^{4}\right.$ and $\left.10^{5}\right)$ as a function of $\tilde{u}$. The inset shows the data collapse for the rescaled function $\frac{D^{\prime}(\tilde{u})}{N}$ for same values of $N$. 